\title{
Assessment of photoselective vaporization of prostate skills during Urology Objective Structured Clinical Examinations (OSCE)
}

\author{
Yasser A. Noureldin, MD, MSc, PhD,; Mohamed A. Elkoushy, MD, MSc, PhD; Nader Fahmy, MD, FRCSC; ${ }^{*}$ \\ Serge Carrier, MD, FRCSC,; Mostafa M. Elhilali, MD, PhD, FRCSC;, Sero Andonian, MD, MSc, FRCSC, FACS
}

*Department of Surgery, Division of Urology, McGill University Health Centre, Montreal QC; † Department of Urology, Benha University, Benha, Egypt; ${ }^{\circledR}$ Department of Urology, Suez Canal University, Ismailia, Egypt

Cite as: Can Urol Assoc J 2015;9(1-2):e61-6. http://dx.doi.org/10.5489/cuaj.2273

Published online February 9, 2015.

\section{Abstract}

Introduction: We evaluated the use of the GreenLight Simulator (GL-SIM) (American Medical Systems, Guelph, ON) in the skill assessment of postgraduate trainees (PGTs) in photoselective vaporization of the prostate (PVP). We also sought to determine whether previous PVP experience or GL-SIM practice improved performance.

Methods: PGTs in postgraduate years (PGY-3 to PGY-5) from all 4 Quebec urology training programs were recruited during 2 annual Objective Structured Clinical Examinations (OSCEs). During a 20-minute OSCE station, PGTs were asked to perform 2 exercises: (1) identification of endoscopic landmarks and (2) a PVP of a 30-g normal prostate. Grams vaporized, global scores, and number of correct anatomical landmarks were recorded and correlated with PGY level, practice on the GL-SIM, and previous PVP experience. Results: In total, 25 PGTs were recruited at each OSCE, with 13 PGTs participating in both OSCEs. When comparing scores from the first and second OSCEs, there was a significant improvement in the number of grams vaporized (2.9 vs. $4.3 \mathrm{~g} ; p=0.003)$ and global score (100 vs. $165 ; p=0.03)$. There was good correlation between the number of previously performed PVPs and the global score $(r=0.4, p=0.04)$. Similarly, PGTs with previous practice on the GL-SIM had significantly higher global score (100.6 vs. 162.6; $p=0.04$ ) and grams vaporized (3.1 vs. $4.1 \mathrm{~g} ; p=0.04$ ) when compared with those who did not practice on GL-SIM. Furthermore, there were significantly more competent PGTs among those who had previously practiced on the GL-SIM (32.7\% vs. $10.2 \%$; $p=0.009)$. PGY level did not significantly affect grams vaporized or global score $(p>0.05)$.

Conclusion: Performance on the GL-SIM at OSCEs significantly correlated with previous practice on the GL-SIM and previous PVP experience rather than PGY level. Furthermore, there were significantly more competent PGTs among those who had previously practiced on the GL-SIM.

\section{Introduction}

Benign prostatic hyperplasia (BPH) is a common progressive disease in aging men. According to the Canadian Urological Association guidelines on $\mathrm{BPH}$, transurethral resection of the prostate (TURP) remains the gold standard of surgical treatment for men with bothersome moderate to severe lower urinary tract symptoms (LUTS). ${ }^{1}$ Transurethral laser approaches are appropriate and effective treatment alternatives to TURP with lower perioperative complications. ${ }^{1}$ These laser approaches, among other procedures, include holmium laser enucleation of the prostate (HoLEP) and photoselective vaporization of the prostate (PVP). Although these laser approaches are associated with shorter convalescence and lower perioperative morbidity, they are also associated with a steep learning curve. For example, at least 20 mentored cases are required for the urologist to be comfortable with HoLEP. ${ }^{2}$ Hence, the Halstedian concept of "see one, do one, teach one" is no longer valid. In addition, reductions in postgraduate trainee (PGT) working hours together with increased public demand for patient safety have created the need for assessment and training of urologists on virtual reality (VR) simulators outside of the operating room. ${ }^{3} \mathrm{~A}$ recent Cochrane review has shown that trainees who exercise on VR simulators perform better in the operating room with less errors and shorter operative time. ${ }^{4}$ In addition, incorporation of VR simulators into the curricula of urology training programs may shorten the learning curves for novice trainees. ${ }^{3}$ One such VR simulator for the PVP procedure is the GreenLight-SIM (GL-SIM) simulator (American Medical Systems, Guelph, ON). ${ }^{5}$ Face, content, and construct validities of this simulator have been recently demonstrated. ${ }^{5-7}$ However, there are no studies regarding the assessment of urology trainees' PVP skills.

The aim of the present study was to incorporate the GL-SIM during urology OSCEs to assess PVP skills of urology PGTs and secondarily to determine whether previous 
Noureldin et al.

PVP experience or GL-SIM practice improved performance on the GL-SIM during OSCEs. We hypothesize that PGY level, previous PVP experience and practice on the GL-SIM will significantly affect performance on the GL-SIM during OSCEs.

\section{Methods}

The GL-SIM is the first high-fidelity simulator for the $532 \mathrm{~nm}$ PVP procedure (Fig. 1). ${ }^{5}$ The simulator scores participants on their success in performing the PVP procedure and provides feedback in terms of grams vaporized and a global score (out of 1000) (Fig. 1). ${ }^{5}$ The simulator automatically calculated grams vaporized and a global score for each PVP procedure performed. Unfortunately, the methods of grams vaporized and global score calculation are proprietary and the manufacturer has not published them.

\section{Study design}

After obtaining Ethics Board approval from McGill University (No: A09-E72-12A) and written informed consents, urology PGTs in PGY-3 to PGY-5 from all 4 urology training programs in Quebec were recruited to participate.

Each OSCE consists of 15 stations of 20 minutes each, with varying number of oral, written, and visual recognition stations. During the two OSCEs of the study (December

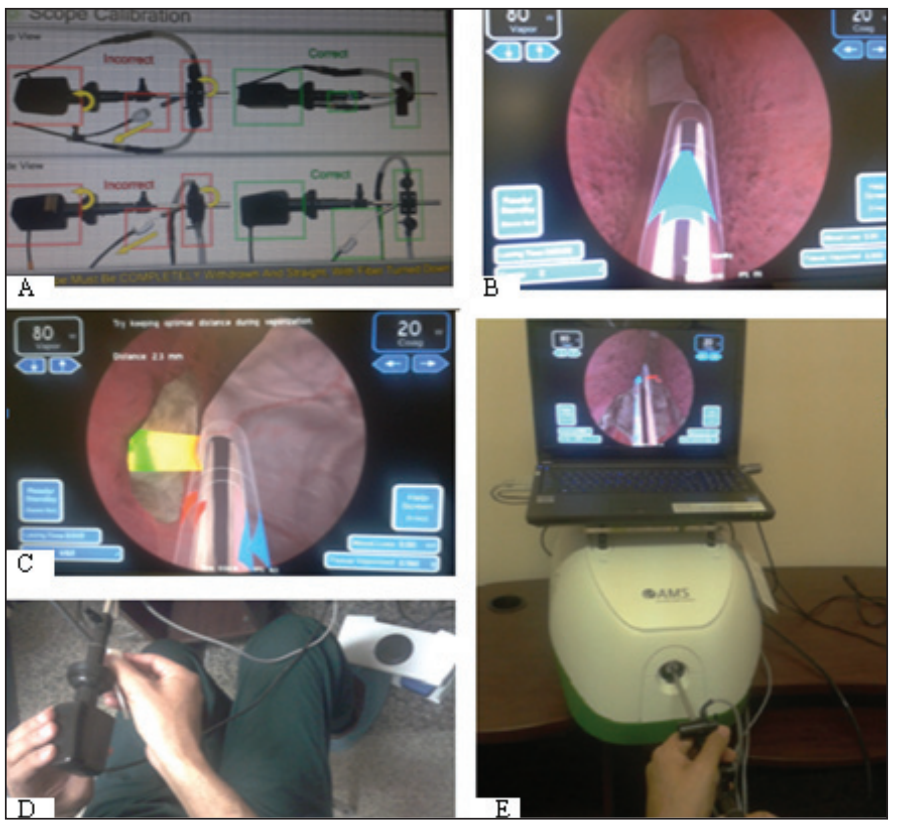

Fig. 1. The GreenLight-SIM Simulator (GL-SIM) (American Medical Systems, Guelph, ON). (A) Diagrammatic representation of the GL-SIM cystoscope and laser fibre. (B) Virtual reality images of the laser fibre and bilateral prostatic lobes protruding into the prostatic urethra. (C) Activated laser fibre that demonstrates the proper distance between the laser fibre and tissue. (D) Position of both hands on the simulator cystoscope and laser fibre in addition to the simulated foot pedal. (E) Simulator attached to a computer.
1, 2012 and December 7, 2013), one of the stations was used to assess the PVP skills of PGTs using the GL-SIM. Participation in the study was voluntary; each participant had the chance to read and sign the consent. Once a participant chose to participate, he was asked to complete a questionnaire regarding age, gender, PGY level, and number of prior cystoscopies, transurethral resection of bladder tumours (TURBTs), TURPs, and PVP procedures performed. In addition, previous practice on the GL-SIM and experience with video games and musical instruments were recorded. After completing the questionnaire, each participant received the same orientation before starting their exercise. Therefore, regardless of their level of training all trainees had the same amount of time to perform two exercises on the GL-SIM: (1) to identify 8 anatomical landmarks and (2) to perform PVP procedure of a 30-g prostate within a 20-minute OSCE station.

At the end of each OSCE, performance scores, including the number of correctly identified anatomical landmarks, grams vaporized and global scores were obtained from the computer software connected to the simulator. All data were coded and subjected to multivariate analysis to determine significant predictors of trainees' performance on the GL-SIM. Performance of PGTs on the GL-SIM was kept confidential from the program director and was not used for promotion purposes. The norm-referenced method was used to determine a competency cut-off score for the global score (the expert group mean minus 1 standard deviation

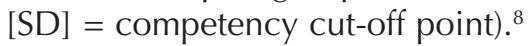

\section{Statistical analysis}

Statistical analysis was done using the Statistical Package of Social Sciences for Windows (SPSS, Chicago, IL) version 20. Descriptive data were presented in terms of number, percentages, means, and standard error of mean. Categorical variables were compared using Fisher's exact test while continuous variables were compared by Mann-Whitney $U$-test and Kruskal-Wallis test or student $(t)$ test and oneway ANOVA test, whenever appropriate, with significance detected at two-tailed $p$ value $<0.05$. Paired comparisons were performed using the Wilcoxon matched pairs signed rank test. Links between continuous variables were measured by the Spearman rank correlation coefficient. Multivariate general linear model was performed for correction of any possible confounders.

\section{Results}

There were no PGTs who declined to participate in our study. Therefore, 37 PGTs, representing the 3 urology training years (PGY-3 to PGY-5) from all 4 urology training programs in Quebec, were included in the study. Since senior 
PGTs in the first OSCE were promoted on July 1, 2013, there were 13 PGTs who attended both OSCEs, rendering the total number of participants in the study to 50 (25 PGT in each OSCE) (Table 1).

When comparing scores from the first and second OSCE, there was a significant improvement in the number of grams vaporized $(2.9 \pm 0.2$ vs. $4.3 \pm 0.4$ g; $p=0.003)$ and global score $(100 \pm 15$ vs. $165 \pm 26 ; p=0.03)$ (Table 1$)$. Furthermore, the 13 PGTs who attended both OSCEs significantly improved their number of grams vaporized from $2.9 \pm 0.3$ to $5.2 \pm 0.5 \mathrm{~g}(p=0.001)$ and global scores from $107 \pm 21$ to $219 \pm 31(p=0.003)$ from the first to the second OSCE.

As expected, the more senior trainees had performed significantly higher number of cystoscopies, TURBTs, TURPs, and PVPs (Table 2). However, there was no significant difference in grams vaporized and global scores among the different years (PGY-3 vs. PGY-4 vs. PGY-5) (Table 2).

There were 13 (52\%) and 14 (56\%) PGTs who had previously practiced on the GL-SIM prior to the first and second OSCEs, respectively. Although all urology programs had access to the KTP laser, only 2 of the 4 programs had access to the GL-SIM. When compared with PGTs without previous practice, PGTs who had practiced on the GL-SIM had significantly higher global scores $(100.6 \pm 19.6$ vs. $162.6 \pm 22.4$; $p=0.04)$ and grams vaporized $(3.1 \pm 0.27$ vs. $4.1 \pm 0.36$; $p=0.04$ ) (Fig. 2). Similarly, there was good correlation between the number of previously performed PVPs and the

\begin{tabular}{lccc}
\hline \multicolumn{3}{l}{ Table 1. Demographics and performance of PGTs in both } \\
OSCEs & $\begin{array}{c}\text { First OSCE } \\
\text { (25 PGTs) }\end{array}$ & $\begin{array}{c}\text { Second OSCE } \\
\text { (25 PGTs) }\end{array}$ & p value \\
\hline Variable & $28.9 \pm 0.4$ & $28.8 \pm 0.6$ & 0.9 \\
\hline Age (years) & $16(64 \%): 9$ & $17(68 \%): 8$ & 1.0 \\
Gender (Male: Female) & $(36 \%)$ & $(32 \%)$ & \\
PGY level & $7(28 \%)$ & $9(36 \%)$ & 0.7 \\
PGY-3 & $9(36 \%)$ & $7(28 \%)$ & \\
PGY-4 & $9(36 \%)$ & $9(36 \%)$ & \\
PGY-5 & $13(52 \%)$ & $14(56 \%)$ & 1.0 \\
PGTs with previous & $430 \pm 74$ & $393 \pm 69$ & 0.7 \\
GL-SIM experience & $85 \pm 27$ & $59 \pm 10$ & 0.4 \\
Cystoscopies (n) & $36 \pm 9$ & $32 \pm 8$ & 0.7 \\
TURBT (n) & $2.8 \pm 1.2$ & $4.5 \pm 1.6$ & 0.4 \\
TURP (n) & $2.9 \pm 0.21$ & $4.3 \pm 0.4$ & 0.003 \\
PVP (n) & $100 \pm 15$ & $165 \pm 25$ & 0.03 \\
Grams vaporized (g) & $5.04 \pm 0.3$ & $5.2 \pm 0.3$ & 0.7 \\
Global score & & & \\
Correct anatomical & identification & & \\
\hline
\end{tabular}

OSCE: Objective Structured Clinical Examinations; PGTs: postgraduate trainees; PGY: postgraduate year; TURBT: transurethral resection of bladder tumour; TURP: transurethral resection of the prostate; PVP: photoselective vaporization of the prostate.

Data are presented in mean \pm standard error or number $(\%)$. global score $(r=0.4, p=0.04)$ (Fig. 3). On average, PGTs had performed 2.8 and 4.5 PVP cases $(p=0.4)$ prior to the first and second OSCEs, respectively.

The norm-referenced method was used to determine a competency cut-off score for the global score. Based on the scores of 3 PVP experts who performed the same exercises within a 20-minute session, the mean global score was $155.33 \pm$ SD 25.54. Therefore, the competency cut-off was $129.79(155.33-25.54=129.79)$. Using this cut-off global score of 129.79 , competency rates among PGTs were then calculated (Table 2, Fig. 4). There were significantly more competent PGTs among those who had previously practiced on the GL-SIM (32.7\% vs. $10.2 \% ; p=0.009)$ (Fig. 4). However, PGY level and previous PVP experience did not significantly affect competency or pass rates (Table 2, Fig. 4).

Regarding the effect of playing video games, there was no significant impact of having prior experience of playing video games on PVP performance parameters in terms of the grams vaporized $(p=0.3)$ and global score $(p=0.1)$. Also students who played musical instruments did not demonstrate significantly higher performance regarding the grams vaporized $(p=0.06)$ and global score $(p=0.1)$ compared with non-players.

\section{Discussion}

Due to decreased trainee work hours and increased public demand for patient safety, there is a need for training on VR simulators to obtain basic procedural skills prior to assisting in the operating room. The role of training on VR simulators is to enhance performance in the operating room with less errors and shorter operative time. ${ }^{4}$ In addition, assessment of basic surgical skills has already become part of the certi-

\begin{tabular}{|c|c|c|c|c|}
\hline Variable & $\begin{array}{l}\text { PGY-3 } \\
(\mathrm{n}=16)\end{array}$ & $\begin{array}{l}\text { PGY-4 } \\
(n=16)\end{array}$ & $\begin{array}{c}\text { PGY-5 } \\
(n=18)\end{array}$ & $p$ value \\
\hline Cystoscopies & $234.7 \pm 65.5$ & $383.8 \pm 79$ & $591.7 \pm 90$ & 0.02 \\
\hline TURBT & $15.5 \pm 5.9$ & $59.5 \pm 6.8$ & $133.3 \pm 33.8$ & 0.009 \\
\hline TURP & $5.3 \pm 1.4$ & $31.7 \pm 6$ & $62.8 \pm 13.8$ & 0.001 \\
\hline PVP & $0.2 \pm 0.2$ & $2.1 \pm 0.7$ & $5.4 \pm 8.1$ & 0.001 \\
\hline $\begin{array}{l}\text { Grams } \\
\text { vaporized }(\mathrm{g})\end{array}$ & $3.4 \pm 0.3$ & $3.6 \pm 0.5$ & $3.8 \pm 0.4$ & 0.7 \\
\hline Global score & $112.4 \pm 28.4$ & $139.8 \pm 22.8$ & $145.4 \pm 29.3$ & 0.7 \\
\hline $\begin{array}{l}\text { Correct } \\
\text { anatomical } \\
\text { identification }\end{array}$ & $5.2 \pm 0.3$ & $5.1 \pm 0.2$ & $5.1 \pm 0.5$ & 0.9 \\
\hline $\begin{array}{l}\text { Competency } \\
(n, \%)\end{array}$ & $5(31.3 \%)$ & $8(50 \%)$ & $8(44.4 \%)$ & 0.54 \\
\hline \multicolumn{5}{|c|}{$\begin{array}{l}\text { OSCE: Objective Structured Clinical Examinations; PGTs: postgraduate trainees; PGY: } \\
\text { postgraduate year; TURBT: transurethral resection of bladder tumour; TURP: transurethral } \\
\text { resection of the prostate; PVP: photoselective vaporization of the prostate. } \\
\text { Data are presented in mean } \pm \text { standard error. }{ }^{*} \text { Competency: PGTs who achieved the pass } \\
\text { score. }\end{array}$} \\
\hline
\end{tabular}




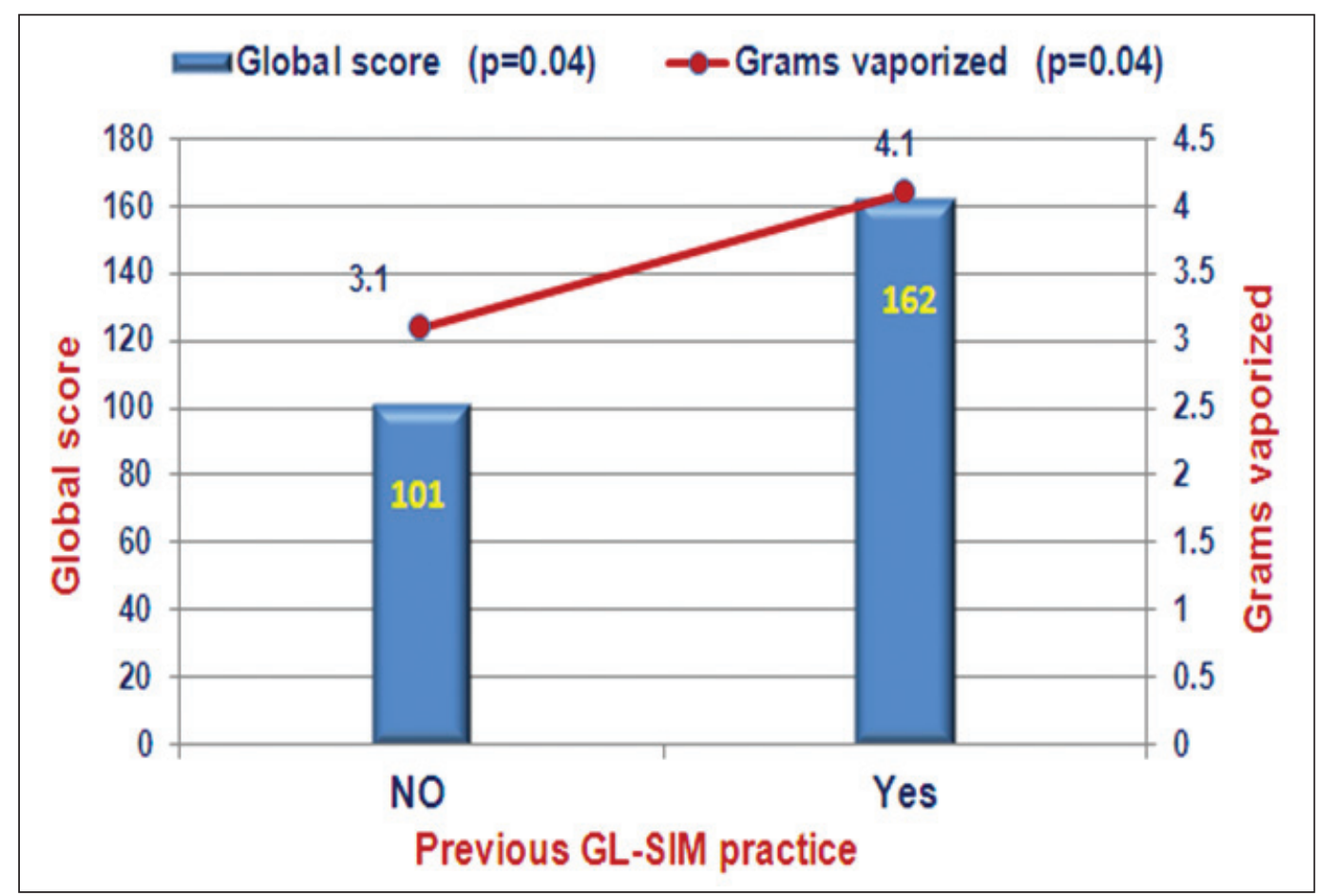

Fig. 2. Effect of practice on GreenLight-SIM simulator on Global Score and Grams Vaporized at Objective Structured Clinical Examinations.

fication process. The American Urological Association has created a Basic Laparoscopic Urologic Surgery (BLUS) skills curriculum based on the Fundamentals of Laparoscopic Surgery (FLS) program. Recently, the transfer task of BLUS was used during OSCEs to assess basic laparoscopic skills of urology PGTs. ${ }^{9}$ In that study, performance on the transfer task of BLUS during OSCEs significantly correlated with the amount of practice rather than the number of laparoscopic cases assisted.

Recently, two studies have confirmed the face, content, and construct validities of the GL-SIM..$^{6,7}$ In the Aydin study, 25 novices consisting of medical students were compared with intermediate level surgeons ( $<70$ PVP procedures) and experts ( $>70$ PVP procedures). However, global scores for training modules were only significantly different between novices and experts. ${ }^{7}$ The other study on the GL-SIM was published by Herlemann and colleagues. ${ }^{6}$ They compared average global scores of 3 trials of 9 novices with 9 experts after 10 weeks of practice on all GL-SIM modules. They found that experts had significantly higher global scores when compared with novices $(836 \pm 11$ vs. $767 \pm 27$; $p=0.008) .{ }^{6}$ Although this was significant, the difference between novices and experts was small $(836-767=69)$. Furthermore, the authors did not record grams vaporized. In the present study, although grams vaporized and global scores increased from PGY-3 to PGY-4 to PGY-5, the differences were not significant $(p>0.05)$ (Table 2). There are several reasons why a significant difference was not seen.
First, PGTs who were recruited for the present study were considered intermediates (performed $<70$ PVP procedures). In addition, since each OSCE station was only 20 minutes, each trainee did not have enough time to finish the PVP procedure. Therefore, our PGTs' global scores were lower than other studies. ${ }^{6,7}$ Furthermore, there was a wide variation in the PVP experience of PGY-5 trainees as evidenced by the large SE $(5.4 \pm 8.1)$ (Table 2). Nevertheless, in the present study there was good correlation between the number of previously performed PVPs and the global score among urology PGTs $(r=0.4, p=0.04)$ (Fig. 3$)$. This is similar to the findings in the Herlemann study. The correlation coefficient $(r)$ in the present study was 0.4 compared with 0.9 in the Herlemann study. The higher correlation coefficient $(r)$ found in the Herlemann study could be related to inclusion of only best scores of experts. ${ }^{6}$

When comparing the scores from the first to the second OSCE, grams vaporized and global scores were significantly better (Fig. 1). This could be due to significant improvements of the 13 PGTs who participated at both OSCEs. In addition, this could also be due to trainees practicing on the GL-SIM to prepare for the OSCE, although the type of simulator used at each OSCE is kept confidential. Similarly, PGTs with previous practice on the GL-SIM had significantly higher scores and grams vaporized when compared with those who did not practice on GL-SIM (Fig. 2). Furthermore, there were significantly more competent PGTs among those who had previously practiced on the GL-SIM (32.7\% vs. $10.2 \%$; 


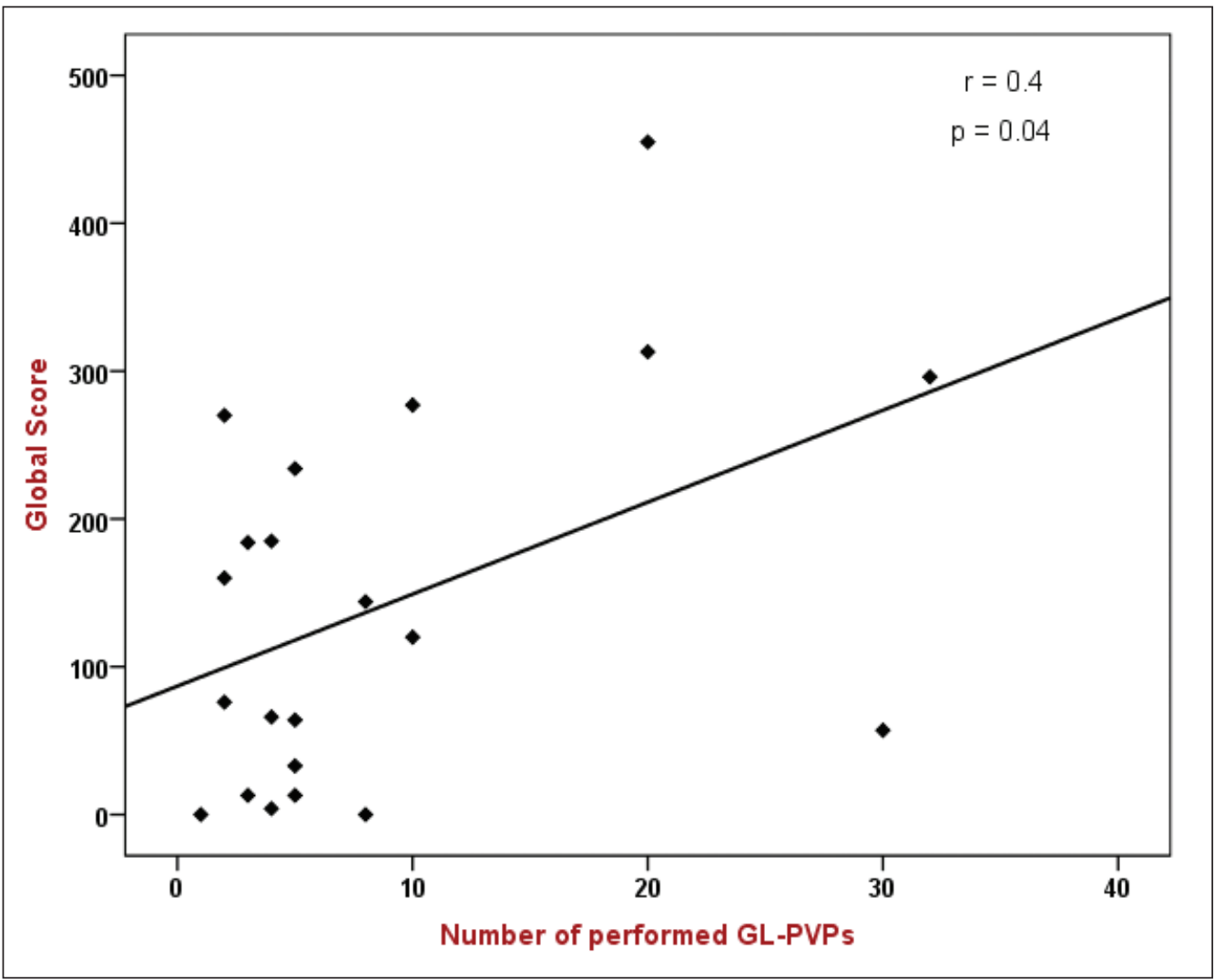

Fig. 3. Correlation between the number of previously performed photoselective vaporization of the prostate and global score.

$p=0.009$ ). This highlights the importance of practicing on the GL-SIM. This is consistent with Erikson's theory on the acquisition of expert performance based on the number of hours spent in deliberate practice..$^{10}$ It is interesting that in the Herlemann study, although participants were given 10 weeks of practice prior to the study, the authors did not report any data whether practice improved their performance. ${ }^{6}$ Similar to the present study, the Aydin study training of novices (medical students) resulted in significantly better global scores with each successive session, with the learning curve levelling after the third practice session. ${ }^{7}$ One added advantage of practicing on the GL-SIM simulator is the fact that trainees do not need to use laser goggles which impede vision. However, the use of KTP laser goggles during actual PVP cases is an important safety measure since several cases of blindness have been reported with improper eye protection. ${ }^{11}$

There is controversial evidence regarding previous experience with video games and musical instruments and their role in improving surgical skills. In the present study, trainees with previous exposure to video games and musical instruments did not have significantly higher scores. In the Herlemann study, participants who played a musical instrument scored significantly higher, barely reaching sig- nificance $(p \leq 0.05) .{ }^{6}$ Furthermore, previous video-gaming did not affect GL-SIM performance. In fact, Kennedy and colleagues studied the effects of video-gaming on the psychomotor, visuospatial, and perceptual abilities in surgical trainees. They concluded that video-gaming positively correlated only with psychomotor rather than visuospatial and perceptual abilities, which are important in urological practice. ${ }^{12}$ However, Van Dongen and colleagues found that playing video games during childhood did not result in superior psychomotor skills in adulthood required for endoscopic surgery. ${ }^{13}$

Despite being a prospective study, this study has its limitations. First, the time limit of the OSCE station was 20 minutes. This may have been too short to vaporize enough prostatic tissue and subsequently obtain higher global scores. In addition, the amount time spent practicing on the GL-SIM was not collected. Another limitation is the relatively small sample size of trainees despite recruiting all urology PGTs from all 4 urology training programs in Quebec. Nonetheless, this is the first study calculating competency cut-off score for the PVP procedure performed on the GL-SIM during a 20-minute OSCE station. Future studies need to recruit PGTs on national or international level to obtain adequate sample size to determine whether 


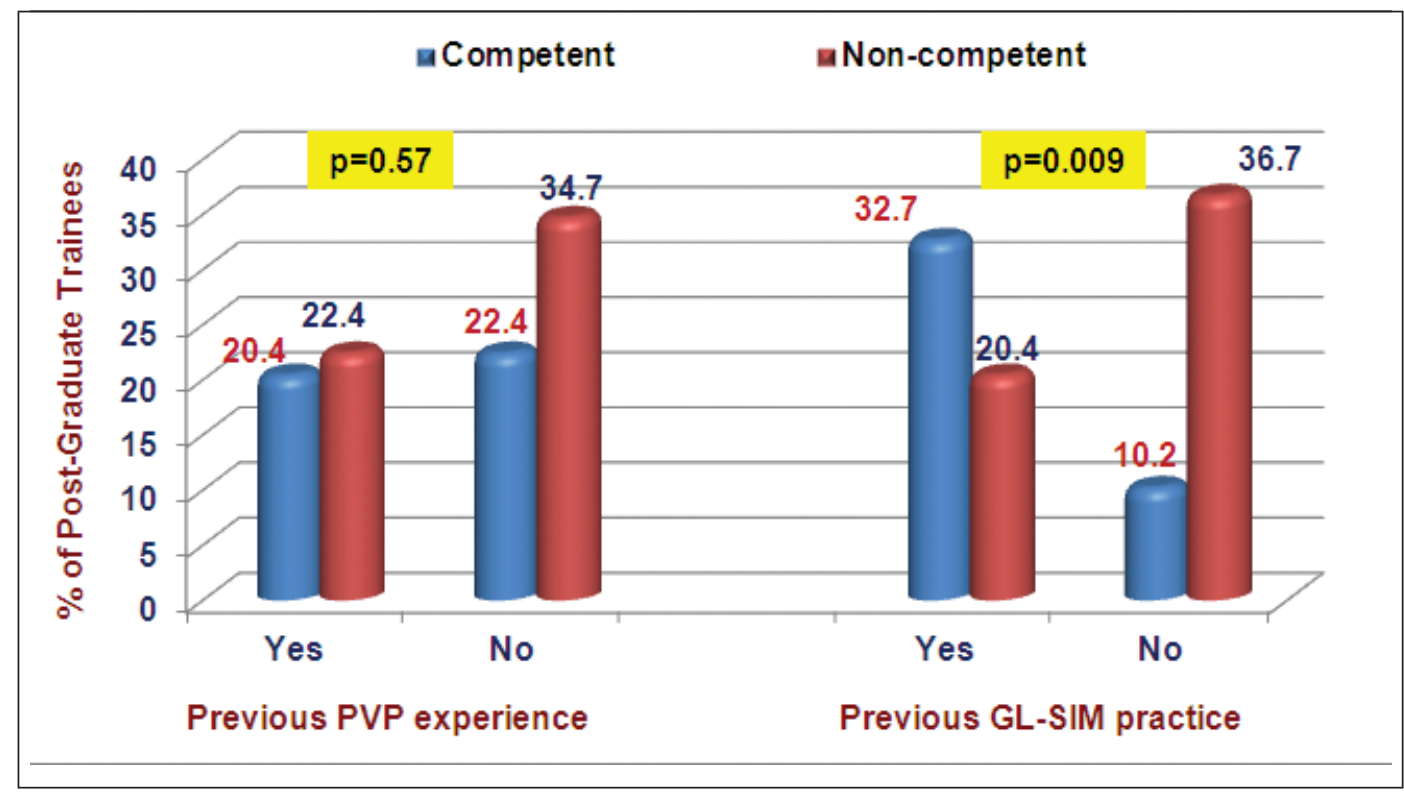

Fig. 4. Effects of previous photoselective vaporization of the prostate experience and previous GreenLight-Simulator practice on the competency of postgraduate trainees during Objective Structured Clinical Examinations.

significant differences exist among different PGY levels using this competency cut-off global score.

\section{Conclusion}

Performance on the GL-SIM at OSCEs significantly correlated with previous practice on the GL-SIM and previous PVP experience rather than PGY level. Furthermore, there were significantly more competent PGTs among those who had previously practiced on the GL-SIM. Future studies need to examine the impact of GL-SIM training on performance during real-life PVP procedures in the operating room.

Acknowledgements: This work was partially sponsored by Fonds de la Recherche Santé du Québec (FRSQ) Chercheur Boursier grant to Sero Andonian. The GL-SIM was provided gratuitously by AMS.

Competing interests: Authors declare no competing financial or personal interests.

This paper has been peer-reviewed.

\section{References}

1. Nickel JC, Méndez-Probst CE, Whelan TF, et al. 2010 Update: Guidelines for the management of benign prostatic hyperplasia. Can Urol Assoc J 2010;4:310-6.
2. El-Hakim A, Elhilali MM. Holmium laser enucleation of the prostate can be taught: The first learning experience. BJU Int 2002;90:863-9. http://dx.doi.org/10.1046/i.1464-410X.2002.03071.x

3. Wignall GR, Denstedt JD, Preminger GM, et al. The surgical simulation: A urological perspective. J Urol 2008;179:1690-9. http://dx.doi.org/10.1016/i.juro.2008.01.014

4. Gurusamy KS, Aggarwal R, Palanivelu L, et al. Virtual reality training for surgical trainees in laparoscopic surgery. Cochrane Database Syst Rev 2013;8:CD006575.

5. Shen Y, Konchada V, Zhang N, et al. Laser surgery simulation plafform: Toward full-procedure training and rehearsal for benign prostatic hyperplasia (BPH) therapy. Stud Health Technol Inform 201 1;163:574-80.

6. Herlemann A, Strittmatter F, Buchner A, et al. Virtual reality systems in urologic surgery: An evaluation of the GreenLight Simulator. Eur Urol 2013;64:687-8. http://dx.doi.org/10.1016/i.eururo.2013.06.008

7. Aydin A, Muir GH, Graziano ME, et al. Validation of the GreenlightTM simulator and development of a training curriculum for photoselective vaporisation of the prostate. BJU Int. 2014; Epub 7 Dec. http:// dx.doi.org/10.1111/bju. 12842

8. George S, Haque MS, Oyebode F. Standard setting: Comparison of two methods. BMC Med Educ 2006;6:46. http://dx.doi.org/10.1186/1472-6920-6-46

9. Elkoushy MA, Luz MA, Delisle J, et al. Determinants of performance on the Transfer Urologic Surgery (BLUS) Curriculum Administered at Objective Structured Clinical Examinations. J Endurol 2013;27:114853. http://dx.doi.org/10.1089/end.2013.0065

10. Ericsson KA. The acquisition of expert performance: An introduction to some of the issues. In: Ericsson KA, ed. The Road to Excellence: The Acquisition of Expert Performance in Arts and Sciences, Sports, and Games. Mahwah, NJ: Lawrence Erlvaum Associates; 1996 :1-50.

11. Althunayan MA, Elkoushy MA, Elhilali MM, et al. Adverse events resulting from lasers used in urology. J Endourol 2014;28:256-60. http://dx.doi.org/10.1089/end.2013.0451

12. Kennedy AM, Boyle EM, Traynor 0 , et al. Video gaming enhances psychomotor skills but not visuospatial and perceptual abilities in surgical trainees. J Surg Educ 2011;68:414-20. http://dx.doi.org/10.1016/i. isurg.2011.03.009

13. Van Dongen KW, Verleisdonk EJ, Schiiven MP, et al. Will the Playstation generation become better endoscopic surgeons? Surg Endosc 2011;25:2275-80. http://dx.doi.org/10.1007/s00464-010-1548-2

Correspondence: Dr. Sero Andonian, Associate Professor of Urology, McGill University Health Centre, 687 Pine Ave. West, Suite S6.92 Montreal, QC H3A 1A1; sero.andonian@muhc.mcgill.ca 